\title{
Event Generator for Particle Production in High-Energy Collisions *
}

\author{
A. Schälicke, T. Gleisberg, S. Höche, S. Schumann, J. Winter, \\ F. Krauss, and G. Soff, \\ Institut für Theoretische Physik, TU Dresden, 01062 Dresden, Germany
}

\begin{abstract}
Event generators are an indispensable tool for the preparation and analysis of particle-physics experiments. In this contribution, physics principles underlying the construction of such computer programs are discussed. Results, within and beyond the Standard Model of particle physics, obtained with a new event generator [1] are presented. This generator is capable to describe signal processes for exotic physics and their backgrounds at electron-positron and proton-(anti)proton colliders.
\end{abstract}

Key words: Hadron colliders, Jets, Standard Model, Supersymmetry phenomenology, Large Extra Dimensions

$P A C S: 13.85 .-\mathrm{t}$

\section{Introduction}

In current collider experiments the hard interaction of two incoming beams results in the production of up to thousands of outgoing particles. So far, no evidence has been found that contradicts the belief that this process is described by the Standard Model (SM) for strong and electroweak phenomena. Unfortunately, a full quantum-mechanical treatment is out of reach. There are two reasons for this apparent shortcoming: first of all, the sheer number of particles involved gives rise to a tremendous number of interfering contributions that grows factorially with the number of particles. Furthermore, perturbation theory is not able to account for the transition of partons to hadrons. This failure of perturbation theory necessitates other methods. Simulation tools called Monte Carlo programs or event generators have proved to be successful

* Talk given by G. Soff at the 25th Course of the "International School on Nuclear Physics" (Erice, September 2003) 


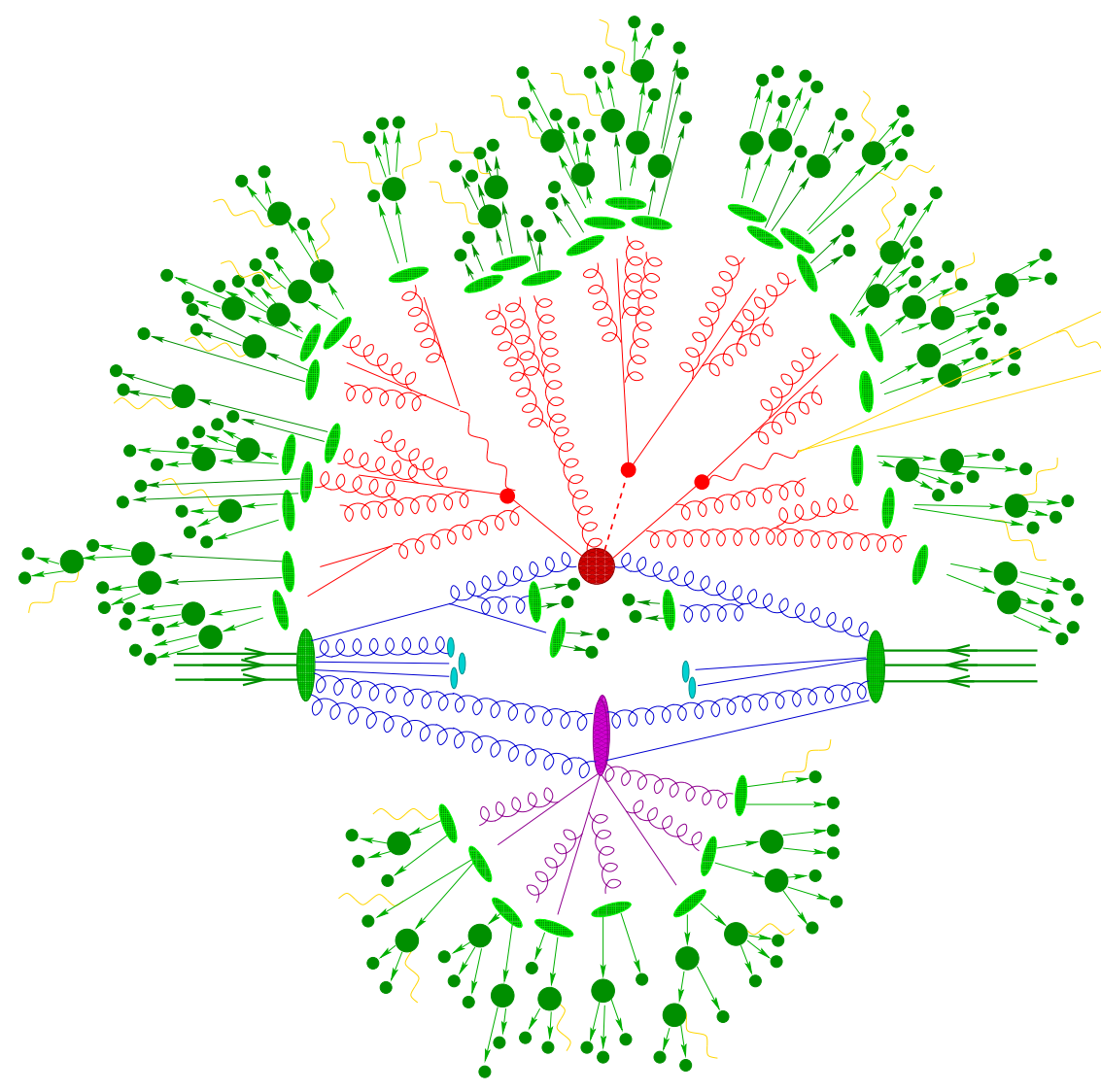

Fig. 1. Sketch of a proton-proton collision from an event-generator point of view.

for a detailed description of multiparticle production. In this contribution we want to briefly illuminate the principles underlying such programs, and we would like to present some results of our new event generator SHERPA [1].

\section{Physics in Event Generators}

For a brief discussion of the physics concepts underlying event generators, Fig. 1 will be employed, in which a proton-(anti)proton collision is sketched. The simulation of such an event has to be decomposed into different independent stages. To control this independence, these stages are ordered according to the scales related to them.

At the highest scale the signal process takes place leading eventually to the production of heavy and/or exotic states. In the figure, this is represented by the thick blob in the middle, in which two gluons fuse to produce a fourparticle final state. For such signals one would like to use the full matrix element. Within our event generator the module AMEGIC++ [2] takes care of the hard processes. It automatically creates all Feynman diagrams, stores them as helicity amplitudes into library files and uses these libraries when the integral over the phase space of final-state particles, i.e. the cross section, is evaluated 
through Monte Carlo methods.

As the event evolves down to lower energy scales, multiple emission of secondary particles must be described. The bulk of radiation activity is in the soft and/or collinear phase-space region, where the interference of subsequent emissions is of subleading logarithmic order only. The logarithms are in ratios of the actual parton-emission scale compared to a suitably defined harder scale, so that, for a description of the radiation pattern, these interferences can be safely neglected. Hence multiple parton emission factorizes into chains of individual parton branchings. Consequently, one obtains a simulation in terms of a parton shower. Radiation off the initial state is more involved, but must result in a boost only, which, due to recoil effects, may also produce some net transverse momentum of the final state. In our event generator, the parton showers are performed by the module APACIC++ [3], which resums all leading logarithms and uses the invariant masses of the decaying partons to order subsequent emissions. An ordering in successive opening angles to properly account for quantum coherence effects is supplemented. A novel feature of our code is that, for the first time, an algorithm has been developed combining matrix elements and the parton shower by smoothly filling the parton-emission phase space and by respecting the logarithmic accuracy [4].

Some particles produced in the hard interaction might be unstable. If their mass is large enough, their decay might involve particles emitted with large energies or under large opening angles. Such decays, again, are treated by using matrix elements and their subsequent parton showers. At high energies the possibly perturbative interaction of the beam remnants, sometimes also coined multiple parton interaction, comes into play. These interactions may develop their own parton showers. However, this phenomenon has the potential to severely obscure the interpretation of experimental data and, therefore, has to be simulated. These two aspects are currently being implemented.

The gauge structure of QCD leads to a breakdown of perturbation theory at scales of the order of $\Lambda_{\mathrm{QCD}}$. So, the parton shower has to stop at such scales and its parton ensemble has to be translated into hadrons, i.e. white objects. Since confinement has not been completely understood yet, hadronization effects have to be modelled phenomenologically introducing parameters that have to be fitted to data. Successful models, such as the string and the cluster model, rely on the fact that before the non-perturbative transition the parton shower has a definite colour structure ${ }^{1}$. Our code incorporates a newly developed cluster-hadronization model and an interface to the well-known Lund string fragmentation, allowing for systematic studies of model uncertainties.

\footnotetext{
1 The parton shower is inherently in a large- $N_{\mathrm{C}}$ approximation, where $N_{\mathrm{C}}$ is the number of colours. Employing a forced splitting of gluons into $q \bar{q}$ pairs, the partons form a set of colour singlets, each of which contains a quark and an antiquark. In cluster models usually formulated in terms of formation and decay of clusters these singlets are interpreted as primordial hadron resonances with indefinite mass decaying into primary, specified hadrons.
} 


\section{Results}

\subsection{Results obtained with AMEGIC++: the parton level}

Here, we will highlight the abilities of AMEGIC++ in calculating cross sections for processes within and beyond the SM. There is a plethora of reasons to search for new physics, e.g.:

The SM has two intrinsic problems, the hierarchy and the naturalness problem, which are addressed to the question why the scale of electroweak symmetry breaking and the Planck scale, where strong gravitational effects are expected, are separated by a wide desert. To understand this hierarchy self-interactions of the (scalar) Higgs boson are considered. Using a cut-off procedure for the ultraviolet divergencies with the cut-off given by the highest scale in the SM, the Planck mass, one immediately realizes that the counter term for the Higgs mass has to be quadratic in the Planck mass. In reality, however, unitarity of $W W$ scattering forces the Higgs mass to be lower than roughly $1 \mathrm{TeV}$. The difference of roughly seventeen orders of magnitude requiring an extreme finetuning (naturalness problem) lets the SM seem somewhat inconsistent.

On top of this intrinsic problems, gravitation is not incorporated by the SM. Assuming the existence of a "theory of everything", one may conclude that the SM is incomplete and constitutes only an "effective theory".

However, the hope to successfully search for new physics depends on our capabilities to understand and subtract the "old" physics, i.e. the SM. Thus it is crucial for any event generator to provide an optimal simulation of SM effects.

\subsubsection{Multi-particle production in the SM: six-fermion final states}

Despite that six-fermion final states constitute significant backgrounds to signatures for new physics, they provide unique opportunities to study some of the most interesting aspects of the SM in great detail. The most important channels are the production and decay of pairs of top quarks and - if existent - of one or more Higgs bosons, the latter allowing to test the structure of the Higgs potential. If no evidence for a Higgs boson was found at the LHC, the study of quartic gauge-boson couplings is mandatory in order to understand alternative scenarios of electroweak symmetry breaking. To test these complex calculations involving up to $\mathcal{O}\left(10^{4}\right)$ Feynman diagrams, tuned comparisons of different generators are required. Table 1 presents predictions of LUSIFER [5], HELAC/PHEGAS [6] and AMEGIC++ [2] for some six-fermion final states corresponding to the production and decay of a top-quark pair. More extensive comparisons of results obtained by HELAC/PHEGAS and AMEGIC++ also taking into account finite fermion masses can be found in [7].

\subsubsection{Cross sections for Supersymmetry}

The ability of our code for searches of new physics is exemplified by some processes within the framework of the MSSM - the Minimal Supersymmet-

ric extension of the Standard Model, see e.g. [8]. Supersymmetry is one of 
Table 1

\begin{tabular}{|l||c|c|c|}
\hline$\sigma[\mathrm{fb}]$ & LUSIFER & HELAC/PHEGAS & AMEGIC++ \\
\hline$\nu_{e} e^{+} e^{-} \bar{\nu}_{e} b \bar{b}$ & $5.853(7)$ & $5.866(9)$ & $5.879(8)$ \\
$\nu_{e} e^{+} \mu^{-} \bar{\nu}_{\mu} b \bar{b}$ & $5.819(5)$ & $5.822(7)$ & $5.827(4)$ \\
$\nu_{\mu} \mu^{+} \mu^{-} \bar{\nu}_{\mu} b \bar{b}$ & $5.809(5)$ & $5.809(5)$ & $5.809(5)$ \\
$\nu_{\mu} \mu^{+} \tau^{-} \bar{\nu}_{\tau} b \bar{b}$ & $5.800(4)$ & $5.798(4)$ & $5.800(3)$ \\
$\nu_{\mu} \mu^{+} d \bar{u} b \bar{b}$ & $17.171(24)$ & $17.204(18)$ & $17.209(9)$ \\
\hline
\end{tabular}

Comparison of tree-level predictions corresponding to six-fermion top-quark pairproduction channels at $E_{\mathrm{cm}}=0.5 \mathrm{TeV}$ for massless fermions; see [9].

the most promising candidates for physics beyond the SM, since it cures the hierarchy problem in a natural fashion: it predicts at least one superpartner for each particle, a bosonic (fermionic) partner for a fermion (boson), with identical quantum numbers and mass. The only difference is in the spins, which differ by one half. In loops, the partners come with opposite sign, thus the quadratic divergencies mentioned before are cancelled. However, no superpartner has been found yet. Hence, Supersymmetry must be broken. The demand to solve the hierarchy problem implies a soft breaking, i.e. the form of breaking terms is limited. First, one usually considers a minimal Supersymmetry, i.e. one superpartner per particle of the SM, and this MSSM is broken by adding all possible breaking terms.

The complete MSSM has been implemented in AMEGIC++, and an example is presented in Fig. 2: gaugino-pair production at an $e^{+} e^{-}$collider shows a strong dependence on the masses of the sleptons propagating in the t-channel. This is true for the lightest charginos $\left(\chi_{1}^{ \pm}\right)$and neutralinos $\left(\chi_{1}^{0}\right)$. Starting with the mSUGRA point ${ }^{2}$ SPS1a [10], the appropriate slepton masses have been varied. For the charginos, the rise for large sneutrino mass exhibits the vanishing destructive interference of s- and t-channel diagrams.
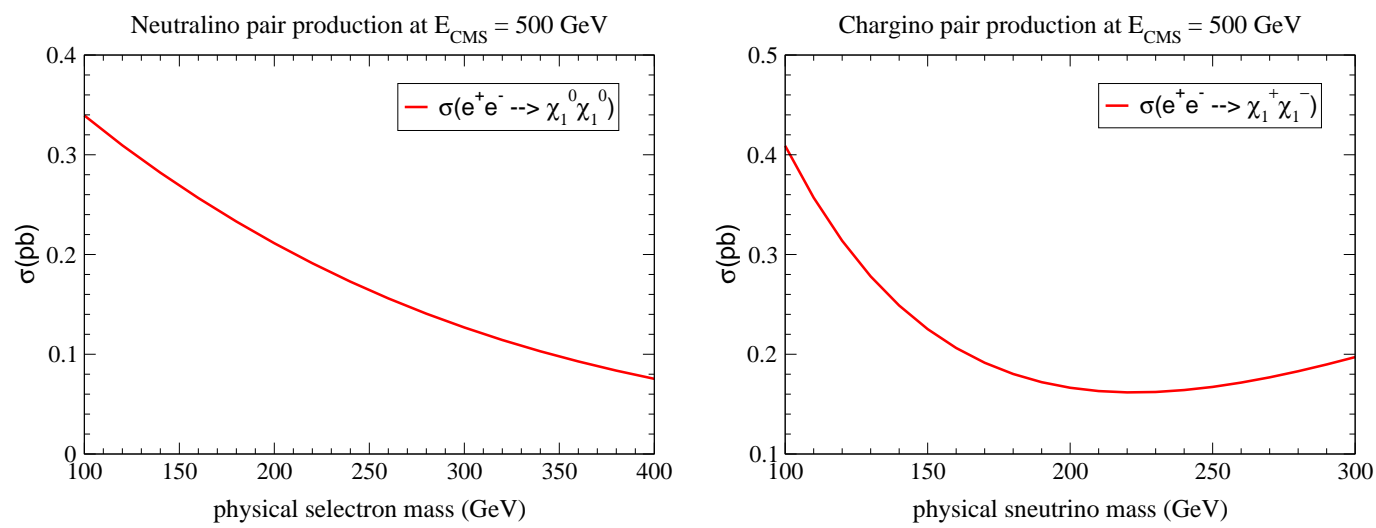

Fig. 2. $E_{\mathrm{cm}}=500 \mathrm{GeV}$ cross sections for $e^{+} e^{-} \rightarrow \chi_{1}^{0} \chi_{1}^{0}$ (left) and for $e^{+} e^{-} \rightarrow \chi_{1}^{+} \chi_{1}^{-}$ (right) in dependence on the selectron and the sneutrino mass, respectively.

$\overline{2}$ For current studies of the phenomenology of the MSSM, specific benchmark points have been proposed [10]. 
Besides $e^{+} e^{-}$physics, a next linear collider might provide an unique opportunity to study $\gamma \gamma$ and $\gamma e$ interactions at high luminosities. Both can be simulated within our event generator. High-energetic electrons are transformed through Compton backscattering of laser light into high-energy photons. This energy spectrum is shown in the left part of Fig. 3. Using the polarization dependence of the backscattered photons, valuable information on particles produced in collisions of polarized beams can be obtained. The right part of Fig. 3 depicts the photon polarization in dependence on its energy-fraction w.r.t. the incoming electron. Figure 4 illuminates smuon pair production exhibiting a significant dependence on the polarization states of the $\gamma$ beams.
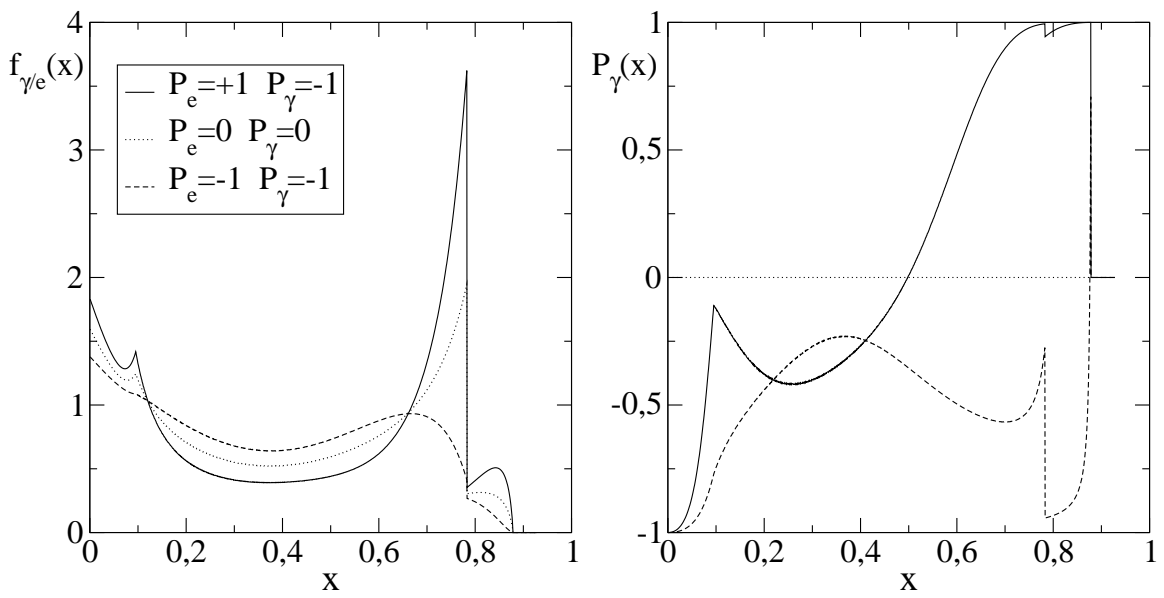

Fig. 3. The photon luminosity spectrum and the degree of circular polarization as a function of the photon energy-fraction $\mathrm{x}=E_{\gamma^{\prime}} / E_{e}$ for different laser and beam helicities in accordance to the parametrization by CompAZ [11], assuming an electron energy $E_{e}$ of $250 \mathrm{GeV}$ and a laser energy $\omega_{\gamma}$ of $1.17 \cdot 10^{-9} \mathrm{GeV}$.

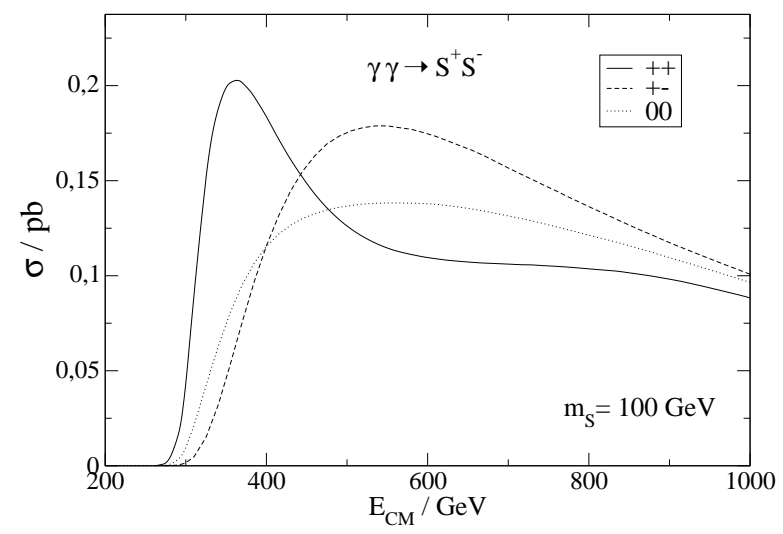

Fig. 4. The total cross section of $\gamma \gamma \rightarrow \tilde{\mu}^{+} \tilde{\mu}^{-}$for $L=0(++), L=2(+-)$ and completely unpolarized beams (00) using the CompAz spectrum, note $\mathrm{S}=\tilde{\mu}$.

\subsubsection{Cross sections for Large Extra Dimensions}

An alternative physics scenario has been recently put forward by ArkaniHamed, Dimopoulos, and Dvali (ADD model) [12]. They propose to solve the hierarchy problem of the Standard Model by shifting the Planck scale down to scales of the order of TeV. This is achieved by introducing $n \geq 2$ new compact 

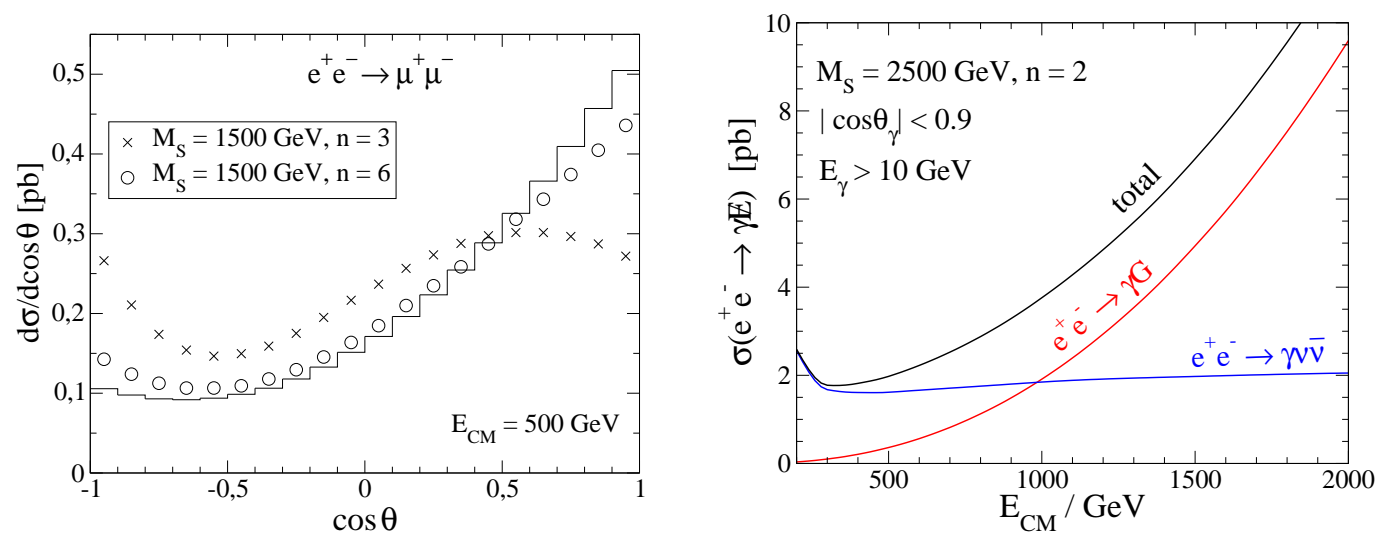

Fig. 5. a) Differential cross section for $e^{+} e^{-} \rightarrow \mu^{+} \mu^{-}$. The solid-line histogram shows the SM background. Crosses and circles depict virtual graviton exchange in two ADD scenarios. b) Total cross section for $e^{+} e^{-} \rightarrow \gamma G$ as a function of $E_{\mathrm{cm}}$.

spatial dimensions that are large compared to the scale of electroweak symmetry breaking. These dimensions can in principle be as large as fractions of millimeters, since only down to these length scales gravity has been probed experimentally. To ensure that the SM remains valid within the regions tested so far, SM particles remain constrained to the common four-dimensional manifold. Only gravitons propagate in all dimension. This changes the laws of gravity and leads to a number of striking signals for the next generation of collider experiments. To study the rich phenomenology of this ADD model, a correct treatment of spin-2 particles in the helicity formalism has been developed and implemented [13]. This allows to describe real as well as virtual graviton production, both of which exemplified in Fig. 5.

\subsection{Results obtained at the hadron level}

They are presented for $e^{+} e^{-}$collisions at the $Z^{0}$ pole, $E_{\mathrm{cm}}=91.2 \mathrm{GeV}$. We will focus on the overall performance of our event generator in its ability to describe multi-hadron production, and on some aspects of our cluster model.

\subsubsection{Overall performance}

First, inclusive observables are considered. This is usually done using eventshape variables, such as thrust, sphericity, oblateness etc., or by looking into the correlations of hadronic matter - jets - deposed in the detector, such as relative angles of jets. In Fig. 6 oblateness and 1-thrust are depicted. The results of our new generator SHERPA [1] are confronted with the results of the well-known PYTHIA Monte-Carlo [14] and with experimental data obtained by ALEPH [15]. Both predictions agree well with the data. Moreover, using DELPHI data [16], Fig. 7 shows the comparison for two more exclusive observables, the $\alpha_{34}$ and the Bengtsson-Zerwas relative angle in four-jet events. There, the impact of our new approach to merge matrix elements and parton showers proves its predictive power. In contrast, PYTHIA adds radiation to the initial two-jet configuration mainly through the parton shower. Therefore, it includes less quantum interference effects. This is the source of the deviation. 

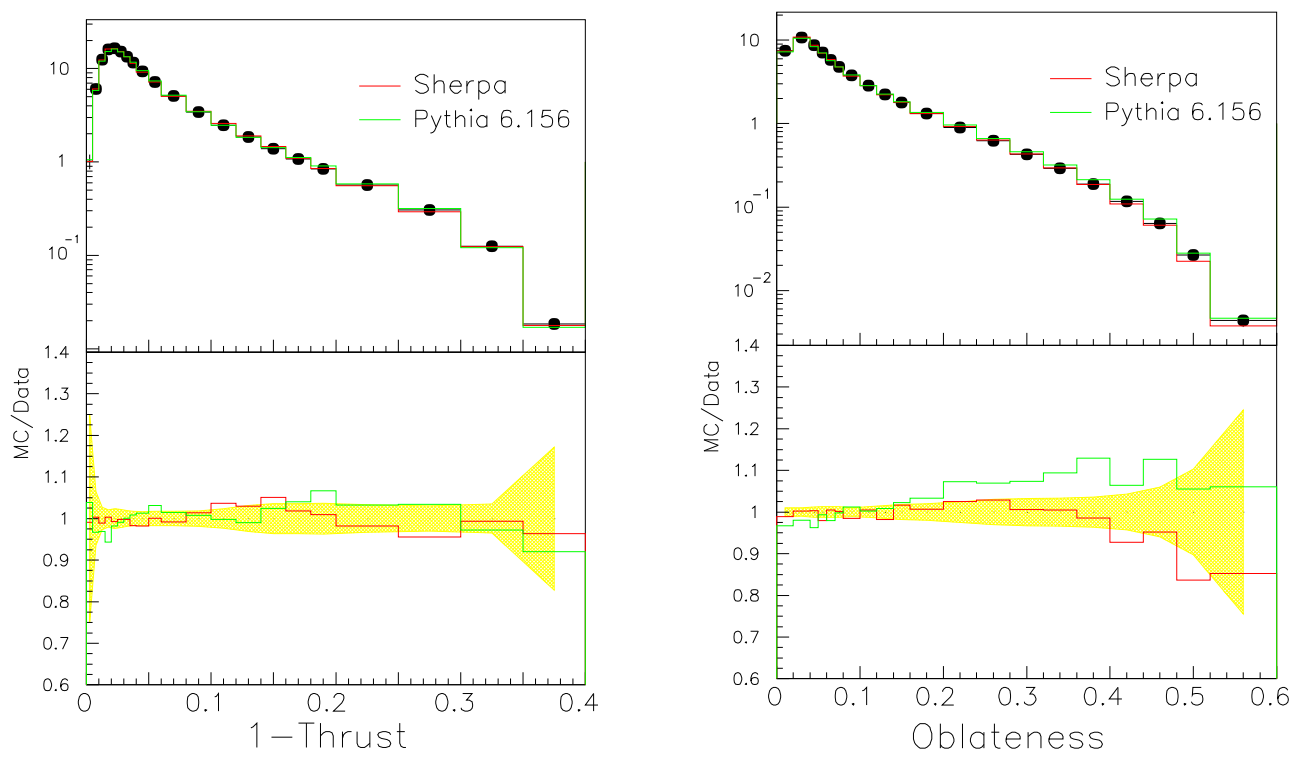

Fig. 6. Event-shape variables for $e^{+} e^{-} \rightarrow$ hadrons at the $Z^{0}$ pole.
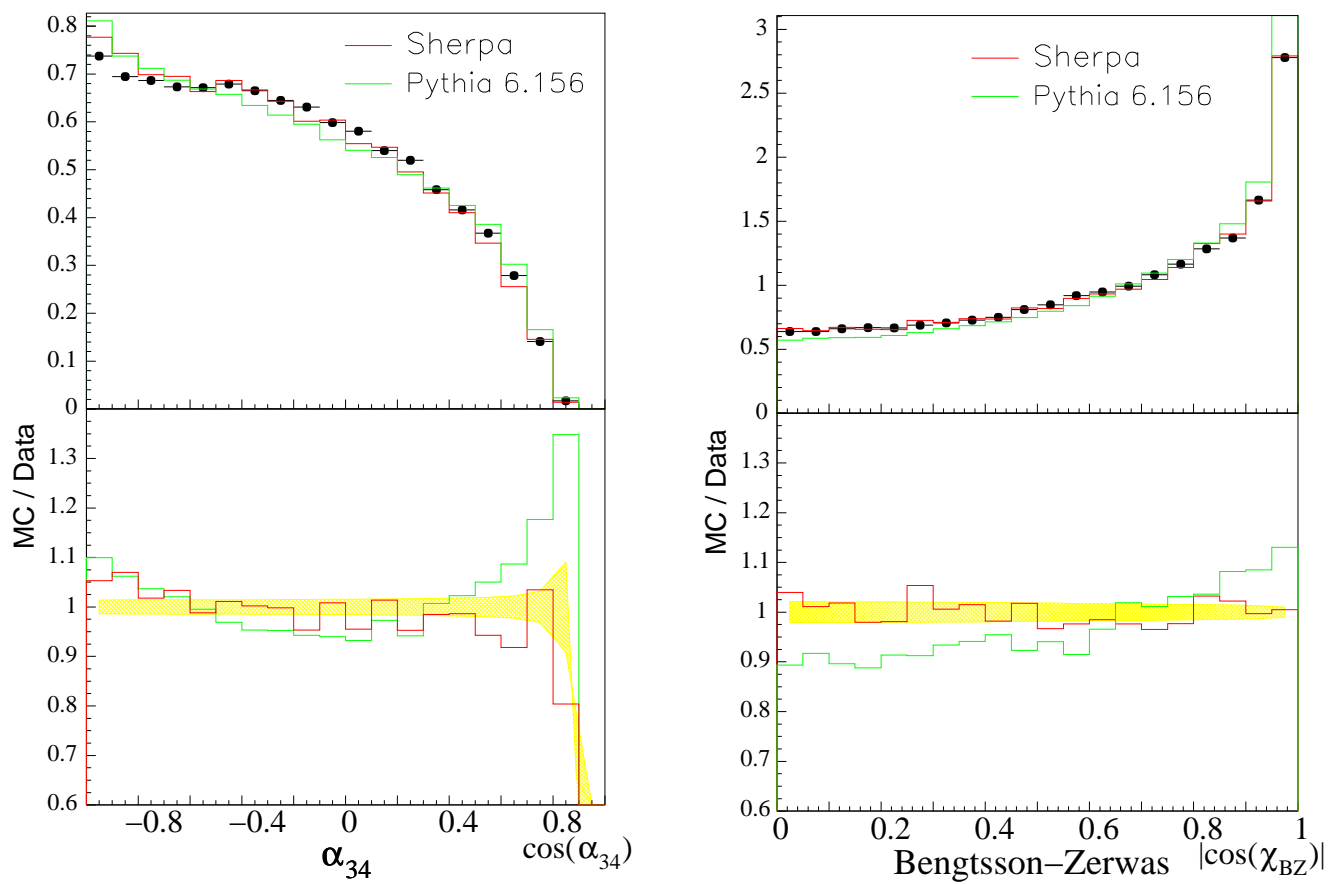

Fig. 7. The $\alpha_{34}$ and the Bengtsson-Zerwas angle [17] for QCD four-jet events.

\subsubsection{Cluster-hadronization model}

Inspired by the well established Webber model [18] for primary hadronization of a coloured partonic system, we have developed a new model presented in [19] and extended by the following features: soft colour reconnection is modelled and incorporated in the formation and fragmentation of clusters, yielding additional cluster-decay configurations. A new mesonic cluster type, the twodiquark cluster, and its decay channels are added. The explicit consideration of the spin of diquark cluster-constituents is rendered possible. The most sig-

nificant feature of our treatment is the flavour-dependent separation of the 


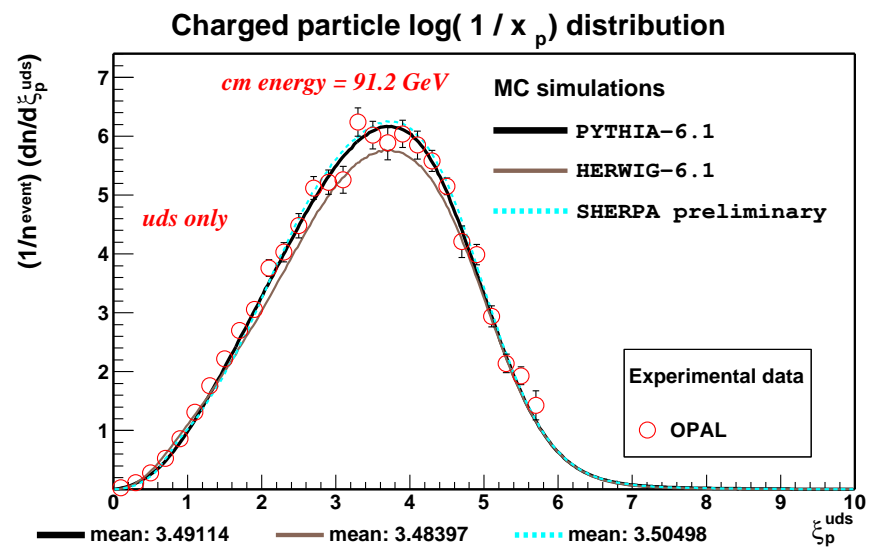

Fig. 8. $\xi_{p}^{u d s}=\ln \left(1 / x_{p}^{u d s}\right)$ distribution of charged particles for $E_{\mathrm{cm}}=91.2 \mathrm{GeV}$ in $e^{+} e^{-}$annihilation into light quarks only. The preliminary SHERPA prediction is presented together with OPAL data [20], and with results from default PYTHIA-6.1 (uds) and default HERWIG-6.1 (uds) [21].

cluster and hadron regimes in terms of the cluster's mass. As a consequence, the different cluster-transition modes are obtained automatically.

A preliminary SHERPA version including our cluster-hadronization model is capable to describe $e^{+} e^{-} \rightarrow \gamma^{*} / Z^{0} \rightarrow d \bar{d}, u \bar{u}, s \bar{s} \rightarrow$ hadrons. The agreement with default PYTHIA-6.1(uds) [14] and experimental light-quark data is satisfactory. To illustrate this, the distribution of the negative logarithm of the scaled-momentum, $\xi_{p}^{u d s}=-\log x_{p}^{u d s}$, is shown in Fig. 8.

\section{Conclusion}

Event generators will continue to be an indispensable tool for future experimental analyses. A new multi-purpose generator for high-energy collisions, SHERPA [1], is currently being developed. It already proved to successfully describe $e^{+} e^{-}$annihilation. The embedded automated matrix-element calculator AMEGIC++ [2] is not only capable to evaluate all Standard Model processes, but also physics beyond the Standard Model (e.g. MSSM and ADD).

The code is written in a transparent structure using the object-oriented programming language $\mathrm{C}++$. Consequently, the implementation of extensions is straightforward; the code is easy to maintain and adjust to the needs of users.

We acknowledge that this work has been supported by BMBF and GSI.

\section{References}

[1] T. Gleisberg, S. Höche, F. Krauss, A. Schälicke, S. Schumann and J. Winter, CERN-TH/2003-284 and arXiv:hep-ph/0311263. 
[2] F. Krauss, R. Kuhn and G. Soff, JHEP 0202 (2002) 044; A. Schälicke, F. Krauss, R. Kuhn and G. Soff, JHEP 0212 (2002) 013.

[3] R. Kuhn, F. Krauss, B. Ivanyi and G. Soff, Comput. Phys. Commun. 134 (2001) 223.

[4] S. Catani, F. Krauss, R. Kuhn and B. R. Webber, JHEP 0111 (2001) 063; F. Krauss, JHEP 0208 (2002) 015.

[5] S. Dittmaier and M. Roth, Nucl. Phys. B 642 (2002) 307.

[6] A. Kanaki and C. G. Papadopoulos, Comput. Phys. Commun. 132 (2000) 306; C. G. Papadopoulos, Comput. Phys. Commun. 137 (2001) 247.

[7] T. Gleisberg, F. Krauss, C. G. Papadopoulos, A. Schälicke and S. Schumann, CERN-TH/2003-282 and arXiv:hep-ph/0311273.

[8] H. E. Haber and G. L. Kane, Phys. Rept. 117 (1985) 75; J. Wess and J. Bagger, Supersymmetry and supergravity, Princeton Univ. Press, Princeton NJ (1992); H. Kalka and G. Soff, Supersymmetrie, B. G. Teubner, Stuttgart (1997).

[9] S. Dittmaier, arXiv:hep-ph/0308079.

[10] B. C. Allanach et al., in "Proc. of the APS/DPF/DPB Summer Study on the Future of Particle Physics (Snowmass 2001)" ed. N. Graf, Eur. Phys. J. C 25 (2002) 113.

[11] A. F. Zarnecki, Acta Phys. Polon. B 34 (2003) 2741.

[12] N. Arkani-Hamed, S. Dimopoulos and G. R. Dvali, Phys. Lett. B 429 (1998) 263.

[13] T. Gleisberg, F. Krauss, K. T. Matchev, A. Schälicke, S. Schumann and G. Soff, JHEP 0309 (2003) 001.

[14] T. Sjöstrand, P. Eden, C. Friberg, L. Lönnblad, G. Miu, S. Mrenna and E. Norrbin, Comput. Phys. Commun. 135 (2001) 238.

[15] R. Barate et al. [ALEPH Collaboration], Phys. Rept. 294 (1998) 1.

[16] H. Hoeth, "Messung der Vierjet-Winkelverteilungen und Bestimmung der QCDFarbfaktoren mit Hilfe des APACIC++-Generators", Diploma Thesis, Fachbereich Physik, Bergische Universität Wuppertal, 2003 [WUD 03-11].

[17] M. Bengtsson and P. M. Zerwas, Phys. Lett. B 208 (1988) 306.

[18] B. R. Webber, Nucl. Phys. B 238 (1984) 492.

[19] J. Winter, F. Krauss and G. Soff, CERN-TH/2003-272, arXiv:hep-ph/0311085; J. Winter, "Formation and fragmentation of hadronic clusters", Diploma Thesis, ITP, Fakultät Mathematik und Naturwissenschaften, TU Dresden, 2003.

[20] K. Ackerstaff et al. [OPAL Collaboration], Eur. Phys. J. C 7 (1999) 369.

[21] G. Corcella et al., arXiv:hep-ph/9912396. 